\title{
Performance Analysis of Discrete Wavelet Multitone Transceiver for Narrowband PLC in Smart Grid
}

\author{
S. Baig and J.Yazdani, Member, IEEE
}

\begin{abstract}
Smart Grid is an abstract idea, which involves the utilization of powerlines for sensing, measurement, control and communication for efficient utilization and distribution of energy, as well as automation of meter reading, load management and capillary control of Green Energy resources connected to the grid. Powerline Communication (PLC) has assumed a new role in the Smart Grid scenario, adopting the narrowband PLC (NBPLC) for a low cost and low data rate communication for applications such as, automatic meter reading, dynamic management of load, etc. In this paper, we have proposed and simulated a discrete wavelet multitone (DWMT) transceiver in the presence of impulse noise for the NB-PLC channel applications in Smart Grid. The simulation results show that a DWMT transceiver outperforms a DFT-DMT with reference to the bit error rate (BER) performance.
\end{abstract}

Index Terms-Discrete Wavelet Transform, OFDM, narrowband PLC, Impulse noise, Smart Grid, automatic meter reading (AMR).

\section{INTRODUCTION}

$\mathrm{R}$ ecent history is witness to tremendous advancement in the fields of digital communication systems and digital signal processing. These progressions have made it possible to utilize the readily available powerlines as a medium of data communication for new emerging applications [1]. Thus, enabling powerlines as a possible contender of broadband data communication, both as an access communication network as well as a local area network for small buildings and in-home networks [2]. The greatest advantage that powerlines hold over the other communication media is their availability as the most pervasive network in the world. Powerlines are accessible even in very remote locations, and offer a "No New Wires" solution to the last mile connectivity problem. However, powerlines are observed as a harsh communication channel, which is plagued by various types of noise [3]. Due to such characteristics, adoption of PLC channel as a broadband data network for high data rate communication has

Sabia Baig is with Electrical Engineering Department, COMSATS Institute of Information Technology, Lahore, Pakistan

(Email: - drsobia@ciitlahore.edu.pk).

Javad.Yazdani is working with the John Tyndall Nuclear Research Institute within the School of Computing, Engineering and Physical Sciences at the University of Central Lancashire, Preston, PR1-2HE, United Kingdom (Email: - jyazdani@uclan.ac.uk). been a debatable issue, despite several successful field trials and tests.

With the explosive increase in energy utilization, the addition of renewable energy resources termed as the "Green Energy" to the power generation and distribution grid seems inevitable [4]. Therefore, there is a strong need to optimize power flow, in order to minimize the wastage of high-cost generation resources and to enhance the utilization of low-cost generation resources. Moreover, automatic meter reading (AMR) and automatic energy management, along with the control, sensing and measurement through powerlines seems to be a promising concept. This idea of utilizing powerlines together with other communication medium enables evolution of the "Smart Grid" [4].

The aging power distribution network has gained a complete new perspective by the emerging concept of Smart Grid (SG). The essential role of communication in the Smart Grid may inherently be assigned to PLC, to act as data carriers for the efficient management of energy distribution [5]. Therefore, in this new and changed scenario, the emphasis has shifted from high cost and more complex broadband PLC techniques towards the low cost and less complex, Narrowband PLC (NB-PLC) techniques [5]. In recent past, NB-PLC application had been restricted to low data rate communication for remote metering and load control. The first generation of NB-PLC modems were based on Frequency Shift Keying (FSK), Binary Phase Shift Keying (BPSK), etc, which offered low data rates up to $5.4 \mathrm{Mbps}$ [6], however, these designs were not able to counter the strong noise content and frequency selective fading PLC channel. NB-PLC got a boost with the introduction of multicarrier modulation techniques, such as the orthogonal frequency division multiplexing (OFDM) and its wire line counterpart, the Discrete Multitone (DMT) modulation technique [2]. Major benefit derived from application of OFDM in PLC was the conversion of frequency selective fading powerline channel to several flat fading subchannels [2]. OFDM has been standardized in the PowerRelated Intelligent Metering Evolution (PRIME) and G3 specifications in the CENELAC-A band (3-95 KHz) [7].

The choice of DMT modems based on discrete Fourier transform (DFT) has some drawbacks, such as the inter-carrier interference (ICI), due to high side-lobes and spectral inefficiency due to the addition of redundant cyclic prefix in the transmit symbols. In this paper authors propose a solution to these problems, as discrete wavelet transform (DWT) based 
DMT modulation technique, namely discrete wavelet multitone (DWMT) for NB-PLC, and present its performance

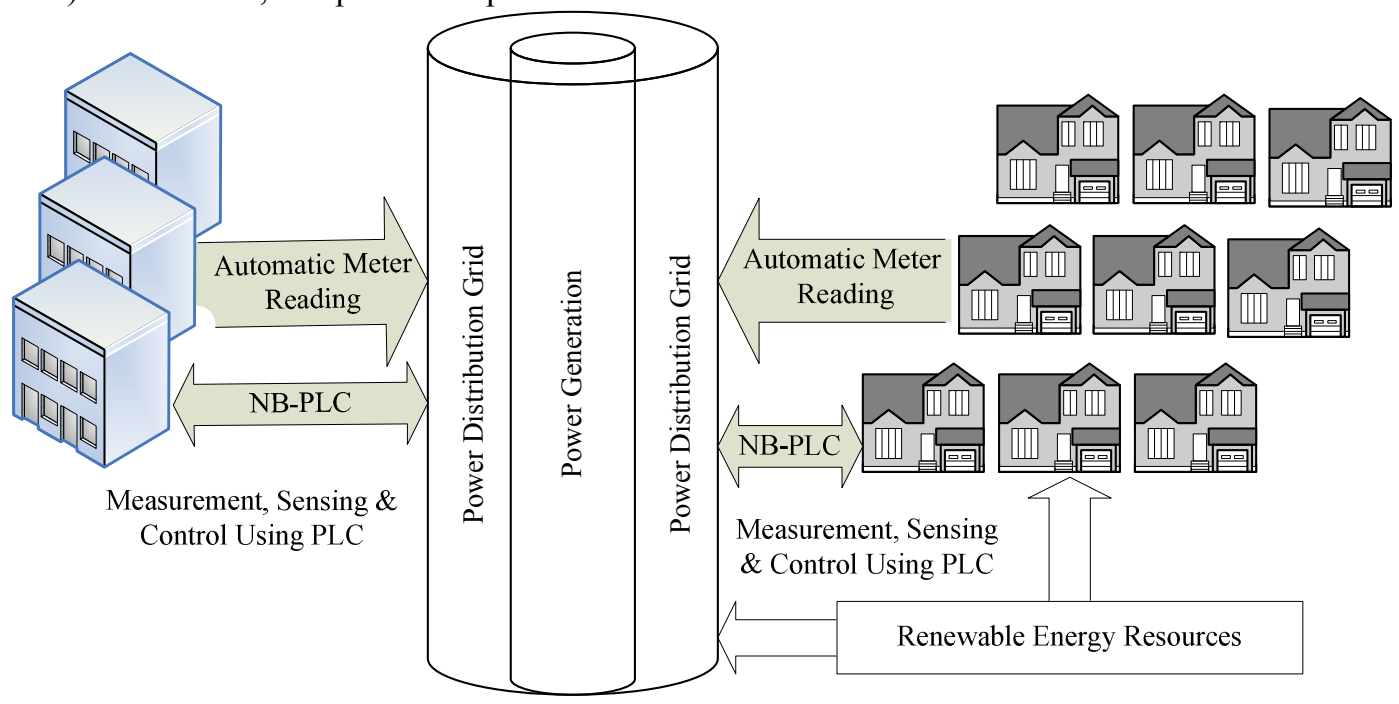

Returning Surplus Energy to the Grid

Fig. 1.Smart Grid Using Narrowband Powerline Communication for measurement, sensing and control

analysis in the presence of impulse noise. DWMT is shown to be a robust modulation technique in comparison to DFT based DMT modulation technique, in the presence of impulse noise. The rest of the article is distributed as follows. Section II discusses the role of PLC, and in particular NB-PLC for SG applications, Section III describes the NB-PLC channel characteristics, including the PLC channel model and the mathematical model of impulse noise. The proposed DWMT transceiver system model is given in Section IV, while Section $\mathrm{V}$ outlines the simulation results, followed by conclusions drawn in Section VI.

\section{ROLE OF POWER LINE COMMUNICATION IN SMART GRID}

Smart Grid (SG) has adopted the role played by Internet, which connects millions of users worldwide for data exchange and communication. However, the SG is the Energy Internet, which unites the functions of power generation and distribution of the electric power grid with automatic measurement, sensing and control functions for intelligent distribution and efficient utilization of electrical energy. For the SG application, the physical infrastructure of power lines is incomplete without the existence of an efficient and robust communication network, in order to facilitate the dynamic flow of energy in response to the fluctuating demand, utilization and rates. Such a cyber-physical system is shown in Fig. 1, which proposes to utilize the low data rate narrowband power line communication for SG applications, like automatic meter reading, etc. Fig. 1 shows that the consumers are able to generate their own power through renewable energy resources, and make use of the two way power flow through the SG. This is made possible with the help of control and communication signals flowing through the power lines, thus facilitating the users to sell the surplus energy to the utility company.

Hitherto, power generation and distribution has been managed through the supervisory control and data acquisition (SCADA). SCADA is the cyber infrastructure model, which performs the role of control and management of the power grid, through human operators, programmable logic controllers, remote terminal units (RTUs) and intelligent electronic devices (IEDs). SCADA relies upon communication media such as wireless radio links, leased lines, dial-up modems, Ethernet, etc. However, SCADA does not support a fully automated system, and requires an operator to control and supervise various operations.

Powerline communication offers an in-built solution to the problem of communication networking, for the SG application, by configuring the physical power delivering infrastructure as a communication network. However, the adoption of powerlines for the purpose of communication in the SG is still a debatable issue, since one group of researchers is in favor of the wireless communication option, while another supports PLC. The ultimate solution may be a hybrid communication network supported by both wireless and wired media. Moreover, the successful deployment of PLC for automatic meter reading (AMR) and automatic metering infrastructure (AMI) in Europe is evidence of the fact that PLC can play a dominant and successful role in the SG communication arena [6]. An obvious advantage of PLC is the already available vast infrastructure, requiring little modification and equipment to convert the grid into a reliable communication network, thereby making its cost comparable to the wireless communication option. Moreover, PLC transceiver may be configured as a communication device or as a sensor depending upon the requirement.

However, PLC in the SG may come across impediments, such as the interference due to interoperability issues between devices operating on various PLC standards. A few PLC standards and applications have been proposed in recent history, for instance, HomePlug 1.0, HomePlug AV, IEEE P1901, etc.

\section{A. High and Medium Voltage PLC Applications for Smart Grid}

PLC application in SG involves communication through the high voltage (HV), medium voltage (MV) and the low voltage 
(LV) power lines. The HV PLC has been recently studied and successfully tested over a $69 \mathrm{kV}$, and $8 \mathrm{~km}$ long line with a data rate of $10 \mathrm{M}$ bps without any repeaters [6]. The HV PLC channel shows little attenuation and time invariant behavior, however, HV lines are affected by noise due to corona. HVPLC can be utilized for remote fault detection, and realtime sag monitoring system of $\mathrm{HV}$ overhead conductors.

MV substations will play a significant role in SG, while transferring information from substation to substation, throughout the grid. PLC can equip these substations with the communication facility without any significant additional cost or equipment. The low speed communication required by the substation IEDs to communicate with the distribution grid IEDs can be through power lines, so as to update the status of fault location, fault isolation as well as for service restoration.

LV PLC is deemed to be the most utilized PLC application for SG communication network. It is discussed in the following subsection.

\section{B. Low Voltage PLC Applications for Smart Grid}

Demand Response (DR) is the methodology of dynamically coordinating the energy supply from generators to meet the load demand, so as to reduce the peak demand spikes. Moreover, the consumers are able to cut down the energy utilization of low priority devices, at the peak demand hours, thereby consuming less energy at high rates. DR is an application of Demand side management (DSM) that utilizes the LV PLC as a communication link, in order to facilitate real-time interaction between generators and loads, for efficient utilization of energy. NB-PLC is more suited for LV PLC, since broadband PLC suffer more from attenuation than NB-PLC.

AMR and AMI make use of NB-PLC for low data rate communication between consumer devices and utility companies. The use of NB-PLC based AMI is more efficient than the wireless solutions, whereby the wireless options, such as Wi-Fi may suffer from network congestion in case many DR signals are trying to access a home device. Therefore, utilization of low data rate communication based on NB-PLC seems advantageous for SG applications, and this paper presents a digital communication solution based on Discrete Wavelet Transform and multicarrier modulation techniques, designed for the harsh and noisy PLC channel.

\section{III. . PLC CHANNEL CHARACTERISTICS}

The PLC channel presents a multipath signal propagation environment, which can be modeled as a linear, frequency selective fading channel, with deep notches in its frequency response. The deep fades are caused by the signal reflections in the cable, due to impedance mismatch [8].Th topology of the low voltage PLC access network between the substation and the customer premises is a star shaped structure, and exhibits lowpass filter characteristic in frequency domain [8]. The high degree of branching in the power grid not only causes reflections, but is also responsible for causing signal attenuation, since each house on the grid absorbs certain amount of transmit power. Moreover, the PLC grid structure varies with the geographic location and therefore it is very difficult to model this channel. Measurements have shown that

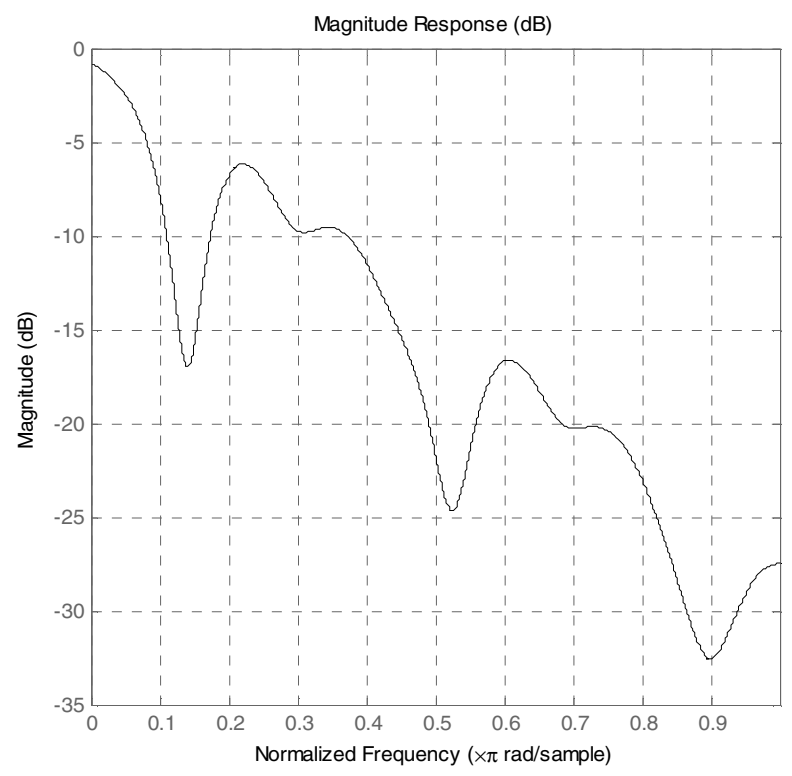

Fig. 2.Powerline channel frequency response

NB-PLC goes through greater fluctuation in impedance below the $500 \mathrm{KHz}$ frequency, as compared to high frequency range, in which the characteristic impedance remains fairly constant [9].

PLC channel is generally assumed to be a quasi-stationary channel. Unlike wireless channel, powerline channel does not alter very fast, and measurements show that its coherence time is large compared with typical symbol duration [10]. Therefore, the NB-PLC channel characteristics are considered stationary, for a transmit symbol duration [11].

\section{A. PLC Channel Model}

Powerlines were originally developed to carry power hence its hostile nature comes from its characteristic impedances embedded in copper line design and as such they were not designed for data communication [12]. PLC channel is classified as a harsh communication channel that exhibits time varying characteristic and hence is difficult to model. Even though there exists no standard PLC channel model, the indoor as well as outdoor powerline channel are in general considered to be multipath frequency selective channel [3].

Zimmermann and Dostert proposed a channel model in parametric form, which represents the low voltage distribution grid as an access network [13]. In [14] authors have classified this channel model for low voltage distribution powerline grid into four categories, according to the length of cable and the number of branches connected to the main cable. The PLC channel transfer function $H(f)$, modeled as $N$ multipaths, is expressed as [13],

$$
H(f)=\sum_{i=1}^{N} g_{i} e^{-\left(a_{0}+a_{1} f^{k}\right)} e^{-j 2 \pi f\left(d_{i} / v_{p}\right)}
$$

where $g_{i}$ is the weighting term, associated with each multipath, $e^{-\left(a_{0}+a_{1} f^{k}\right)}$ is the attenuation term and $e^{-j 2 \pi f\left(d_{i} / v_{p}\right)}$ is the delay term, which defines the time delay 
associated with each path. Fig. 2 shows the channel transfer function for $N=6$ paths, with various parameters defined in [14].

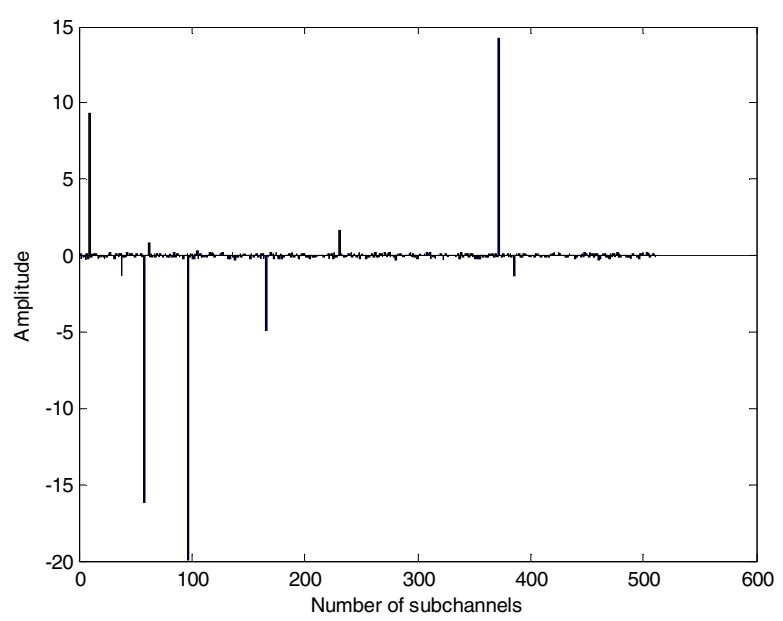

Fig. 3 Simulation of impulse noise

\section{B. Impulse Noise Model}

Powerlines are well known for their noisy communication environment, which mainly consists of background noise and impulse noise [13].The background noise appears to be stationary, while the impulse noise emerges for short intervals with a high power spectral density(PSD) of up to $50 \mathrm{~dB}$ above the background noise [14]. The high magnitude associated with the randomly occurring impulse noise may cause errors in data transmission over powerlines. Impulse noise is generated by the switching transients in the network. Impulses occur for a short time period, varying between a few microseconds to milliseconds. The impulse noise probability density function (pdf) is characterized by the Class A noise, and its probability density function (pdf) for a random variable $x$ is [15],

$$
p(x)=\frac{1}{2 \pi} \sum_{m=0}^{\infty} \frac{1}{\sigma_{m}^{2}} \alpha_{m} \exp \left(-\frac{|x|^{2}}{2 \sigma_{m}^{2}}\right)
$$

where $\alpha_{m}=e^{-A} \frac{A^{m}}{m !}, \sigma_{m}^{2}=\sigma^{2} \frac{(m / A)+T}{1+T}, \sigma^{2}$ denotes the Class A noise variance, $T=\sigma_{g}^{2} / \sigma_{l}^{2}, \sigma_{g}^{2}$ is the AWGN variance, and $\sigma_{l}^{2}$

is the impulse noise variance. $A$ denotes the impulse noise index.

The proposed DWMT transceiver for the NB-PLC is evaluated with Additive White Class A Noise (AWCN) model, which represents the complex, memoryless, additive white Class A noise channel model, computed as a combination of AWGN and impulse noise [15]. The simulated impulse noise is shown in Fig. 3. The impulse noise simulation parameters are defined as, $\sigma_{g}=10^{-3} \mathrm{~W}, T=0.001$ andA is set to 0.001 .

\section{DWMT BASED TRANSCEIVER FOR NB-PLC}

DWMT transceiver is proposed in this paper for NB-PLC, a form of powerline communication, which is specifically suited for Smart Grid applications. The proposed system model is discussed in this Section. Discrete Wavelet Multitone
(DWMT) is the suggested variant of multitone modulation technique based on wavelet or wavelet packet filter banks [16]. Wavelets and multirate filter banks are finding novel applications in the field of digital communications, especially in different areas of powerline communication [17]. This is due to the remarkable properties of wavelet filter banks, which lead to perfect reconstruction without aliasing using non-ideal filter banks, provision of orthogonal basis, multi resolution analysis, etc. DWMT has been proposed for NB-PLC transceiver, so as to mitigate the inter-carrier interference due to the application of DFT filter banks in the DMT modulation based PLC modem.

\section{A. Wavelet Filter Banks}

Wavelets in DWMT serve as an alternative to the exponential basis functions of DFT-DMT. Wavelets are basically small oscillatory waves that formulate a signal transform capable of analyzing non-stationary signals in a joint time-frequency domain. This sort of analysis is not possible with the classical Fourier transform techniques [18]. Therefore, the greatest advantage offered by DWT is that the basic functions of wavelet transforms are localized in both time and frequency.

According to Mallat's algorithm DWT can be implemented using an octave spaced wavelet filter bank [18].Two-channel filter bank that is shown in Fig. 4 can be utilized to construct an octave-spaced filter bank with the help of a tree type structure. In this tree-type structure, the two-channel filter bank consists of a lowpass filter $G(z)$ and a highpass filter $H(z)$ for separating input signal into sub-bands in frequency domain. The lowpass filter $G(z)$ and the highpass filters $H(z)$ are the quadrature mirror filter (QMF) banks, having complementary frequency response. The octave filter bank is constructed by the successive decomposition of the lowpass signal into constituent sub-bands, using the twochannel filter bank [18]. For a wavelet packet filter bank, both the lowpass and the highpass filters are further decomposed into constituent sub-bands repeatedly.

\section{B. DWMT System Model}

The DWMT system model's block diagram is shown in Fig. 5, which splits the input data bit-stream into multiple and parallel bit-streams. The proposed design of DWMT transceiver is based on discrete wavelet packet transform (DWPT), which is implemented through a reverse order perfect reconstruction filter bank transmultiplexer. The blocked version of the input signal is mapped to a variable QAM constellation according to the number of bits loaded, using Water Filling bit-loading algorithm [19]. This is interpolated and filtered by the $k^{t h}$ branchsynthesis filter $F_{k}(z)$ in the IDWT block. The combined transmit signal is communicated through the NB-PLC channel, and the received signal is equalized by a time domain equalizer. The equalized signal is then passed through the corresponding analysis filter $H_{k}(z)$ and decimated to retrieve the QAM encoded version of the transmitted signal. The transmitted signal is recovered after QAM decoding. 


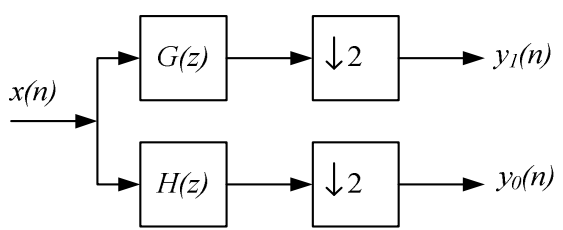

Fig. 4. Two channel Quadrature Mirror Filter bank

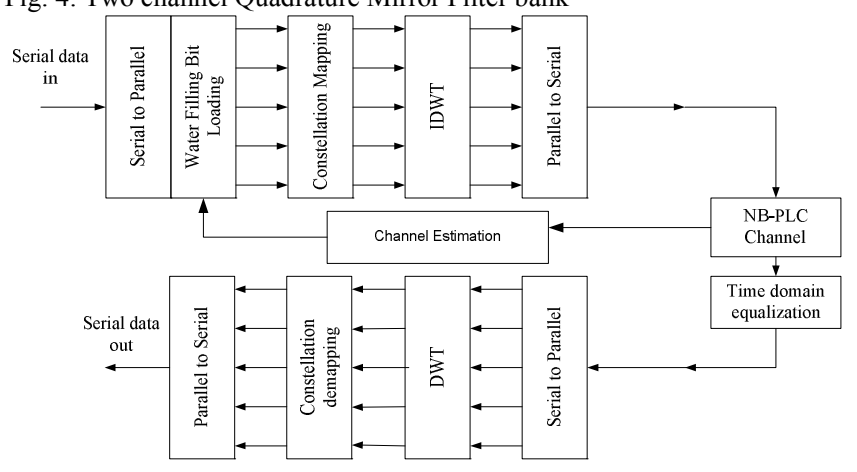

Fig. 5. Discrete Wavelet Multitone System Model for a NB-PLC channel

Water-Filling bit loading algorithm applied in the proposed system is rate adaptive and it is suitable for achieving maximum bit rate and also useful when considering the large number of sub-channels and variable QAM constellation [19].

\section{Simulation RESUlts}

The NB-PLC for the newly envisioned Smart Grid applications, such as AMR requires more reliable and fast communication as is offered by the typical FSK or BPSK based modems. Although, OFDM/DMT based modems are successful in efficient utilization of the spectrum, however these suffer from ICI, due to greater magnitude side lobes. Therefore, DWPT based DWMT transceiver is simulated using MATLAB and its $B E R$ performance is analyzed in a simulated NB-PLC channel with AWGN as background noise. The DWMT transceiver utilizes Water Filing bit loading algorithm to allocate between 1 to 6 bits/sub-channel, in accordance with the sub-channel SNR, as shown in Fig. 6. The proposed system is equalized using minimum mean squared error-decision feedback (MMSE-DFE) time domain equalization technique. $B E R$ performance of the proposed DWMT system is compared with a DMT system in identical channel and noise conditions, as shown in Fig. 7. It is observed that DWMT system shows an improvement of $4.9 \mathrm{E}-$ 5 BER over DMT system's BER of 8.3E-4 at $E_{b} / N_{o}=$ $30 \mathrm{dBs}$.

The $B E R$ performance of the two systems is then comparedfor the NB-PLC channel and AWCN. For this purpose a combination of AWGN and Middleton Class A noise for the impulse noise index $A=0.0001$ is applied [20].DWMT based transceiver gives better $B E R$ performance as compared to its conventional DFT-DMT counterpart, as shown in Fig. 8. It is observed that the overall $B E R$ of both systems has deteriorated in the presence of impulse noise. DWMT system shows an improvement of 7.1E-3 BER over DMT system's BER of $2.5 \mathrm{E}-2$ at $E_{b} / N_{o}=30 \mathrm{dBs}$.

\section{CONCLUSIONS}

Powerlines present a challenging medium for low data rate communication systems in the NB-PLC for Smart Grid applications. Emerging signal processing technologies based on wavelets and multirate filter banks offer unique flexibility for signal analysis, channel characterization, and are indeed superior in mitigating narrowband interference present in the powerline channel. The significance attached to wavelet based OFDM is evident from the fact that it has been standardized for high-speed PLC in the IEEE P1901 PHY layer. The DWMT transceiver's performance analyzed in this paper can be further enhanced with various forms of coding and interleaving techniques.

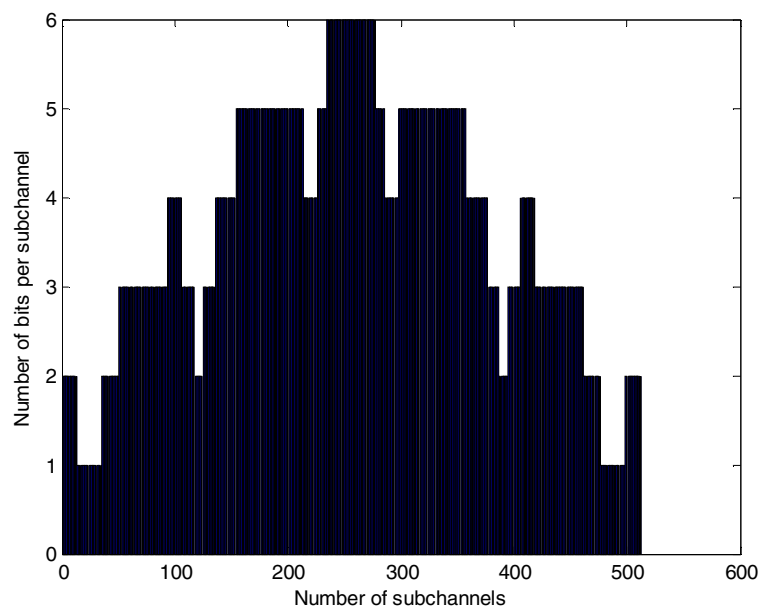

Fig. 6. Bit allocation in accordance with Water Filling bit-loading algorithm for simulated PLC channel

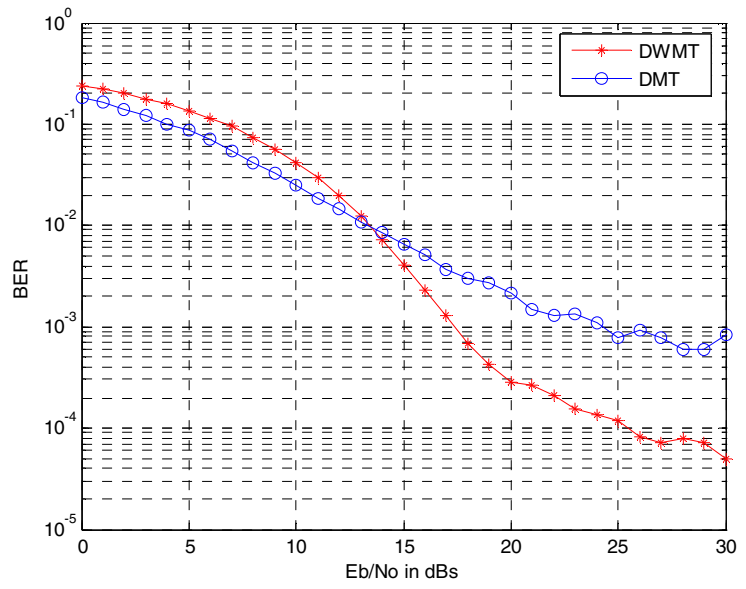

Fig. 7.Bit Error Rate Performance of DWMT vs. $E_{b} / N_{o}$ for NB-PLC 


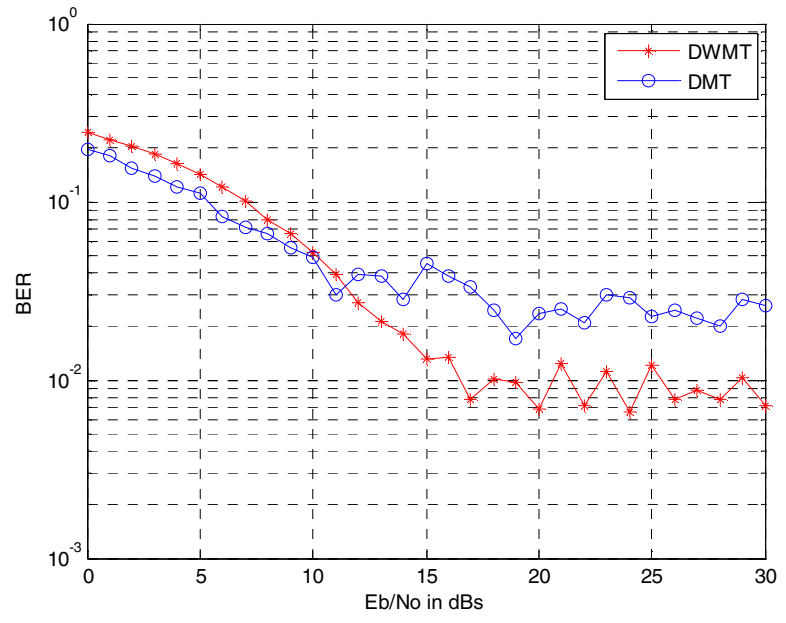

Fig. 8. Bit Error Rate Performance of DWMTvs. $E_{b} / N_{o}$ for NB-PLC with Impulse noise

\section{REFERENCES}

[1] Jianming Liu; Bingzhen Zhao; Jiye Wang; Yi Zhu; Jing Hu, "Application of powerline communication in smart power Consumption," in Procs. OfIEEE International Symposium on Powerline Communications and Its Applications (ISPLC), vol., no., pp.303-307, 28-31 March 2010.

[2] S. Baig and N. D. Gohar, "Discrete Multitone Transceiver at the heart of PHY Layer of an In-Home Powerline Communication Local Area Network ,". IEEE Communications Magazine, vol.41, no.4. pp 48-53, April 2003.

[3] E. Biglieri, "Coding and Modulation for a Horrible Channel,"IEEE Communications Magazine, vol. 41, no. 5, pp. 92-98, May 2003.

[4] Krzysztof Iniewski, Convergence of Mobile and Stationary NextGeneration Networks, New York: Wiley, Chapter 11, Tae Eung Sung, Adam Bojanczyk, Power-line communications and Smart grid SEP 2010.

[5] Galli, S.; Scaglione, A.; Zhifang Wang; "Powerline Communications and the Smart Grid," in Procs. Of IEEE First International Conference on Smart Grid Communications vol., no., pp.303-308, 4-6 Oct. 2010.

[6] Stefano Galli, Anna Scaglione, Zhifang Wang, For the Grid and Through the Grid: The Role of Powerline Communications in the Smart Grid.arXiv: 1010.1973v2 [cs.NI].

[7] Souissi, S.; Ben Dhia, A.; Tlili, F.; Rebai, C.; "OFDM modem design and implementation for narrowband Powerline communication," in Procs. Of 5th International Conference on Design and Technology of Integrated Systems in Nanoscale Era (DTIS), vol., no., pp.1-4, 23-25 March 2010.

[8] M. Gotz, M. Rapp, and K. Dostert, "Powerline Channel Characteristics and Their Effect on Communication System Design," IEEE Communications Magazine, vol. 42, no. 4, pp. 78-86, Apr.2004.

[9] M. Katayama, "Introduction to Robust, Reliable, and High-Speed Power-Line Communication Systems," IEICE Trans. Fundamentals, vol. E84-A, no. 12, pp. 2958-65, Dec. 2001.

[10] H. Philipps, "Modeling of Powerline Communication Channels," in Proc.of IEEE ISPLC, 1999.

[11] Del Re E., Fantacci R., Morosi S., Seravalle R.: "Comparisonof CDMA and OFDM Techniques for Downstream Powerline Communications on Low Voltage Grid", IEEE Transactions onPower Delivery, Vol-18, n. 4, pp. 1104-1109, October 2003.

[12] N. Pavlidou, A. J. Han Vinck, J. Yazdani, and B. Honary. Powerline Communications: State of the Art and Future Trends. IEEE Communications Magazine. 41(4) pp. 34-40. doi:10.1109/MCOM.2003.1193972. April 2003.

[13] M. Zimmermann and K. Dostert, "A multipath model for thepowerline channel," IEEE Transactions on Communications, vol. 50, no. 4, pp. 553-559, 2002.

[14] P.J. Langfeld. "The Capacity of Typical Powerline Reference Channels and Strategies for System Design,"Procs. of the 5th International Symposium on Power-Line Communications and its Applications, ISPLC-2001, Malmö, Sweden, pp. 271-278, April 4-6, 2001
[15] G. Pay and M. Safak, "Performance of DMT Systems under ImpulsiveNoise," in Procs. of International Symposium on Power-Line Communications and Its Applications2001, pp. 109-114 Malmo, Sweden, Apr.2001.

[16] S. D. Sandberg and M. A. Tzannes, "Overlapped discrete multitonemodulation for high speed copper wire communications,"IEEE J. Select. Areas Commun. vol. 13, no. 9, pp. 1571-1585,Dec. 1995.

[17] S. Baig and M. J. Mughal, "Multirate signal processing techniques for high-speed communication over power lines," IEEE Communication Magazine,pp. 70-76, Jan. 2009.

[18] S. Mallat, A Wavelet Tour of Signal Processing, 2nd ed.,Academic, 1999.

[19] Leke, A.; Cioffi, J.M.; "A maximum rate loading algorithm for discretemultitone modulation systems," in Procs. of IEEEGlobal Telecommunications Conference, GLOBECOM '97, vol.3, no., pp.15141518, Nov 1997.

[20] D. Middleton, "Statistical-physical models of electromagneticinterference," IEEE Transactions on Electromagnetic Compatibility,vol. 19, no. 3, pp. 106-127, 1977.

\section{BIOGRAPHIES}

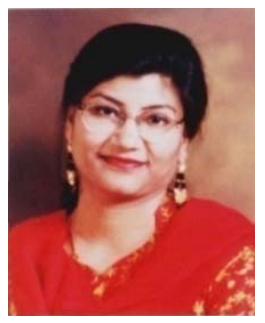

Sobia Baig is heading the MultiRate Communication Networks (MRCN) research group at the Electrical Engineering Department, COMSATS Institute of Information Technology, Lhr, Pakistan. She received her B.Sc. degree in Electrical Engineering from the University of Engineering and Technology, Taxila, Pakistan, in 1995. She did her Masters and PhD from the Faculty of Electronic Engineering, GIKI, Topi, Pakistan in 2003 and 2008 respectively, in the field of Digital Communications of Electronic Engineering. Currently, she's working as Assistant Professor in the Electrical Engineering Department of COMSATS Institute of Information Technology, Lhr. Prior to this; she contributed as Assistant Professor, Faculty of Electronic Engineering, GIK Institute. Her research interests include multicarrier modulation techniques and application of wavelet based modulation techniques in the wireline access networks and power line communication networks.

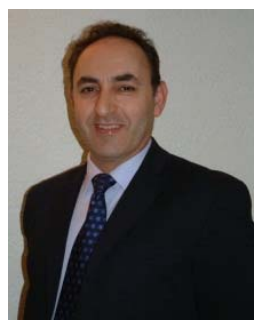

Javad Yazdani is the academic lead for the Smart Grid Technology and Powerline (SGT/PLC) systems group. Javad received his BEng/MEng Honours in Mechatronics Engineering, MSc in Digital Signal Processing (DSP) and $\mathrm{PhD}$ in Communication Systems entitled "High Frequency Digital Power Line Transmission for Terrestrial and Marine Networks" from Lancaster University in 1990, 1996 and 2001 respectively. Javad has been researching in the field of Powerline for almost 17 years with considerable industrial experience in Nuclear Engineering.

Javad is supervising number of MSc and $\mathrm{PhD}$ students and has published many technical papers in the refereed journals and international conference proceedings. His research focus is in the field of Smart Grid related to utilizing PLC as a hybrid communication medium for smart and intelligent sensor/control. Javad is also exploring the role of Smart Grid and grid vulnerability with respect to nuclear power plant security. Javad is a Senior Lecturer in Nuclear Engineering and the Head of uclan|Nuclear undergraduate programmes within the School of Computing, Engineering and Physical Science at the University of Central Lancashire. 